\title{
Guidance of healthcare development for metastatic cancer patients as an example for setting incentives
}

\author{
Jörg Haier ${ }^{1,4}$. Jonathan Sleeman ${ }^{2,3}$. Jürgen Schäfers ${ }^{4}$
}

Published online: 16 December 2019

(c) Springer Nature B.V. 2020

\section{Translating national cancer care targets}

A major challenge and matter of debate is how to break down and translate Universal Healthcare Coverage (UHC) goals effectively into national cancer care targets. To achieve UHC aims, cancer care delivery structures and processes need to be incentivized in an appropriate manner. In a recent editorial we argued that while the incentivizing effects of specific healthcare frameworks can achieve the intended targeted effects, there can also be unintended negative consequences caused by the healthcare framework itself, as well as by misleading or harmful prioritization of secondary individual interests by participants in the healthcare delivery system. These factors are highly dynamic within developing cancer care systems. Since healthcare frameworks for cancer care in low und middle income countries (LMIC) differ significantly from those in high income countries, establishing the right incentives is crucial, and needs to be adapted to the specific conditions in these countries. This is especially true for cancer patients with metastatic disease, due to their increased vulnerability and stronger dependence on specialist care. In this editorial we outline how financing models and insurance schemes impact on the efficacy of incentivization in LMIC, and the consequences this has on cancer care in these countries.

Jörg Haier

haier.joerg@mh-hannover.de

1 Comprehensive Cancer Center, Hannover Medical School, Hannover, Germany

2 Medical Faculty Mannheim, University of Heidelberg, ECAS, Ludolf-Krehl-Str. 13-17, 68167 Mannheim, Germany

3 Karlsruhe Institute for Technology (KIT), Campus Nord, Institut für Toxikologie und Genetik, Postfach 3640, 76021 Karlsruhe, Germany

4 IGP-Institute for Health Sciences and Public Health, Muenster, Germany
Incentives influence individual and organizational behavior. At least in the long-term, they should facilitate provider competition guided by quality measurements, clinical refinement, and economic competition [1]. Their careful design needs to provide an adequate decision-making environment [2]. By applying these principles not only to physicians but also to the entire cancer care system and beyond, incentives at the macro-, meso- and micro-level [3] can improve individual and system effectiveness through better alignment with performance goals. Such incentives can be related to payment systems with the aim of delivering high-quality, cost-conscious UHC care. However, consideration of incentives should not be limited to these aspects alone, since all aspects of UCH can directly or indirectly influence availability, accessibility, acceptability, affordability and quality. Behavioral economics has emerged as a tool for analyzing and developing effective incentives for physicians and other participants within the cancer care system, with the aim of delivering high value care for patients and society. In addition to economic stimuli for providers, other types of behavior-guiding rewards such as various sociocultural incentives are of similar or even greater importance, especially for LMIC. The different types of incentive are summarized in Table 1.

\section{Financing of services and reimbursement}

The simplest financing system for cancer care is a fixed salary for key employees without any adjustment to account for patient numbers, performance, or economic indicators. This payment scheme provides easy management. Furthermore, it may provide an incentive to work in less attractive regions, such as rural and poor areas, assuming that the salary offered is competitive compared to alternative forms of employment. However, fixed salary remuneration tends to result in low performance with regard to the type of patient groups that are covered, the cases that are treated, the number and 
Table 1 Groups of incentives, their types of implementation and levels of benefits

\begin{tabular}{lll}
\hline Groups of incentives & Types of implementation & Level of benefits \\
\hline Financial & Salary & Personal \\
& Infrastructure investment & (Personal or) institutional \\
& Reimbursement & Institutional \\
& Education opportunity & Personal (or institutional) \\
& Research opportunity & Personal or institutional \\
Complexity of management & Institutional \\
& Market access & Institutional \\
& Time availability & Personal or institutional \\
Cociocultural & Housing & Personal \\
& Social recognition & Personal \\
& Political & Personal (or institutional) \\
Access to professional qualification & Personal \\
& Professional recognition & Personal (or institutional) \\
Self-responsibility and decisional power & Personal (or institutional) \\
& Career development & Personal or institutional \\
\hline
\end{tabular}

complexity of procedures used, and the clinical quality that is delivered. This is especially true for vulnerable subpopulations with high treatment requirements, such as advances stage cancer patients with metastatic cancer.

Reimbursement as fee-for-service (FFS) is a common practice in many LMIC countries. Healthcare frameworks may include FFS at the organizational level or as individual salaries/benefits. FFS models and salary-based payments can be more or less problematic depending on the structure and organization of the healthcare system concerned. For example, an insufficient framework of incentives for cancer care can stimulate ineffective system development, including excessive bureaucracy and poor resource management. In these contexts, patients with complex diseases who require multiprofessional and interdisciplinary care, such as those with metastatic cancer, are likely to receive less attention and poorer quality of care, potentially resulting in underdiagnosis and/or undertreatment. Overall, FFS systems have a clear tendency to be deficient in stimulating quality and efficiency. FFS payment schemes may be based on capitation (capped payment per patient for a certain period of medical care delivery), which can positively stimulate acceptance and availability of cancer care structures, especially in underserved regions. On the down side, capitation generally tends to induce inefficient capacity usage, for example by limiting treatment to just the basic requirements in order to increase profitability. For example, cancer patients who require simple procedures (usually those with earlier disease stages) become attractive in such models, whereas advanced cancers with time-consuming treatment are less accepted by cancer care providers in these contexts [4]. Furthermore, financial incentives comparable to capitation, such as variable compensation structures for physicians provided by different types of insurance (public versus private), can significantly predict the guidance of patients towards specific cancer care providers. Lower reimbursement levels by public insurance schemes discourage physicians in private practices from accepting new patients, and highly vulnerable populations, such as patients with advanced cancer, may be at even greater risk of having impaired accessibility to cancer care structures.

Innovation in delivery processes is also generally not well supported under FFS, which is mainly due to the lack of appropriate incentives. Investment in the implementation of innovations, which can be economic, organizational or sociocultural, are not rewarded by capitation systems. Similarly, negative incentives are lacking, such as restricted reimbursement for lower quality of care or the avoidance of complex treatments. This may have secondary effects, since cancer care workers in less innovative environments tend to leave this sector. As a consequence, additional impairment of availability, accessibility and quality of cancer care ensues, which again has the most harmful impact on vulnerable groups of cancer patients such as those with metastatic disease who require specialized care.

In view of these problems, alternatives to FFS payment are becoming more widespread with the aim of improving the value delivered within the cancer care system, using fewer resources to provide the same benefit to all cancer patients, or using equivalent resources to achieve improved cancer care that includes complex treatments. A major challenge of all these alternatives is that they require considerable investment in reporting, analytics, controlling and support systems. The analysis of treatment patterns provides options and motivations for providers to adapt cancer care pathways accordingly. If transparent, the results of these 
analyses can also enable patients to be more engaged in their care, resulting in improved acceptability. Reimbursement systems that do not rely on capitation need to use forms of reporting that are based either on procedures, performance or other targeted values. Such reporting requires valid and reliable data structures, proper reporting pathways and controlling mechanisms in order to achieve the anticipated incentivizing effects. Due to the multidimensional aspects of quality in cancer care, this is particularly difficult to achieve. In addition, the data structure for various targeted values (for example, providing access to palliative care) may differ significantly between countries and FFS models, which also needs to be taken into account. For example, procedurebased reimbursement depends on the grouping of economically comparable procedures and treatments based on the average working time, the requisite resources, the specialization required and structural investments. It is important to recognize that these expenditure-driven data are generally not suitable for quality assurance purposes.

Options to avoid the unwanted consequences of dominant capitation are mixed models that combine capitation with various value-based reimbursements. The incentivizing effects of these mixed models differ between countries and depend on:

a) the dominant form of reimbursement,

b) the extent of procedure-based financing and its coverage of real expenditures,

c) the regional availability of cancer care delivery structures,

d) the allocation of infrastructure (including human resources) to different provider structures,

e) value-based reporting pathways, and

f) the management structures that are available.

Recently, variants of diagnosis-related financing schemes (DRG systems) have become popular in LMIC. These reimbursement models rely on national cost weights that are derived from cost calculations from existing standard hospitals, risk adaptation for comorbidities, socioeconomic aspects and national patient data reporting systems. The major advantage of DRG systems is the stimulus on providers to provide good quality of care and to efficiently use available resources. However, significant risks may be induced by case-related payments, including inadequate patient selection due to the avoidance of risks, in inappropriate focus on economically advantageous treatments that can induce overtreatment, and shortcomings in time-consuming aspects of patient care. However, DRG systems may also provide stimuli for the treatment of vulnerable, advancedstage cancer patients, if the complex care that these patients require is considered appropriately within the grouping of procedures and treatments. Realization of the incentivization potential of DRG systems appears to require compulsory nationwide structures, solid expenditure calculations and adequate reporting structures for DRG-related patient data.

\section{Performance and value based systems}

To overcome the limitations of FFS reimbursement schemes, various pay-for-performance $(\mathrm{P} 4 \mathrm{P})$ models have been implemented in a number of LMIC countries. These models are usually based on special financial payments for a dedicated performance. However, both the structure of the financial rewards as well as the definition of performance (including its quantification and measurement) differs significantly between the models. It is therefore difficult to compare and transfer results between different countries. In general, P4P programs need to acknowledge that incentives have to be valuable enough to stimulate investment in improving cancer care. Their impact relies exquisitely on high-powered incentives that directly target improved cancer care processes and enhanced patient experience, as well as on the availability of achievable benchmarks for improved outcomes.

It is important to recognize that the value of an incentive has to be seen from the perspective of its receiver, which is not necessarily the same as the perception of those who design the respective incentivizing framework. In addition, the perception of incentives can vary over time, as well as between age groups, generations and different professional groups. This necessitates the continuous adaptation of the underlying schemes. P4P incentives need to focus on a limited number of reliable performance indicators to motivate cancer care providers into changing delivery processes, and require a design that enables clinical and organizational leaders to sustainably use the system for performance improvement. This is a highly challenging task, particularly in the context of cancer care. Furthermore, adequate risk adjustment, such as for differences in underlying patient populations (for example, curable cancer stages versus palliative care situations) or catchment structures (for example urban versus fare remote areas), are important design aspects. High impact performance indicators will motivate providers to develop better cancer care and reorganize efficient delivery processes. However, there is always the inherent danger of stimulating untargeted effects, such as the avoidance of patients with clinically complex conditions or economically less attractive care seekers, such as individuals with metastatic cancer stages. Overall, the effective implementation of P4P for patients with cancer, and particularly those with advanced stages, remains an enormous unsolved task, and therefore is currently not recommended for LMIC, as considerable public health research into the implementation $\mathrm{P} 4 \mathrm{P}$ in the cancer care arena needs to be carried out. 
Classic P4P models may be modified or combined with incentives, and applied across entire delivery processes that are mainly used for chronic diseases. For example, disease management programs (DMP) with standardized care for certain cancer entities are a combination of FFS and P4P, which commonly integrate structural, process and outcome parameters. If these programs are used for cancer preventive strategies, such as HPV immunization, population coverage and outreach can be improved. In addition, these shortterm treatment programs may achieve economic effectivity and positive cost-benefit ratios [5]. However, their success is exquisitely related to the availability of resources and the motivation of healthcare providers. Furthermore, the effects achieved by incentivization are determined in part by patients' acceptance of and participation in cancer care structures, and on the cultural and religious environment in which the cancer care is embedded. In addition, the education of both the health care providers (in terms of their professional knowledge) and patients (in terms of their understanding of the provider and access models) impacts on the efficacy of incentivization. Accordingly, counterproductive effects of DMP that worsen inequalities in healthcare delivery may occur, especially in remote regions.

Implementation of DMP and other P4P models is frequently impeded by a variety of barriers. Clinical care processes need to be clearly defined that have a limited need for individualization of care. This means that these models are only suitable for a small number of diseases or medical conditions that require highly standardized and comparable medical approaches, which is not usually the case for cancer. Mechanisms are also required that allow for the dynamic adaptation of care plans based on recent innovations and new clinical evidence, especially if care plans are legally regulated. Furthermore, these models demand high management, reporting and controlling efforts, as well as the definition of reliable and measurable quality indicators. Accordingly, poor availability of the high data quality and data management processes that are required limit implementation of these models.

Overall, implementation of DMP in LMIC for specific chronic diseases and acute intervention programs may provide an opportunity to improve the quality of care through standardization of delivery processes and performance feedback, if implemented as part of the DMP. However, there is also a high risk that quality improvement will fail, and that enhanced accessibility for patients will not materialize. Reasons for this include the complexity of DMP, the need to intensively integrate patients into the program, the high requirements for education of involved staff and patients, as well as the requirement for adequate reporting structures. These issues can also be accompanied by unexpected increased costs. In summary, when the potential risks and benefits for cancer care offered by P4P models are weighed against each other, such approaches cannot be recommended for LMIC.

In addition to the frequently used economically-driven performance parameters incorporated into typical $\mathrm{P} 4 \mathrm{P}$ designs, there is increasing interest in the advantages offered by value-based financing. The primary goal of value-based payment in the management of cancer patients is to achieve improved care that is not immediately measured as an increased delivery performance by cancer care providers, but rather as a better cancer patient experience, improved clinical quality and health outcomes, or lower overall costs of cancer care. In contrast to performance-based financing, the theoretical background of value-based financing includes a less restrictive perspective on achievable advantages, fewer constraints on management decisions, as well as a reduced need for measurement and reporting. Various forms of value-based financing (such as shared savings and risk, reference pricing, and bundled payment), combined with adjunct incentives for quality and efficiency may be tailored according to the prevailing market conditions and organizational frameworks. However, translation of broadly accepted and comparable values into economic categories and reimbursement schemes is highly controversial. For example, there are a number of ethical considerations due to the great potential of value-based financing to increase inequality in population coverage especially for poor people, to worsen cancer care availability in underserved regions, and to impair its acceptability for certain population groups. In addition, assessment of values such as "health outcomes" is widely used, but significant differences in understanding the meaning of such terms exist between healthcare professionals and managers, making universal applicability challenging. To date, little evidence is available concerning the relative effectiveness and underlying mechanisms of value-based purchasing designs compared to other financing systems, including for LMIC. The very limited current experience suggests that value-based systems require complex management, infrastructure and human resources, as well as functioning referral and effective reporting pathways, if adequate UHC is to be achieved. These observations appear to exclude the use of value-based models for cancer care in LMIC, at least in the near future.

Healthcare economists have advocated the use of costeffectiveness analysis (CEA) in several (mainly industrial) countries. The principle of CEA is to rank healthcare interventions on the basis of their incremental costs relative to their incremental benefits. This approach invariably leads to the prioritization of treatments, resulting in inequality of healthcare coverage based on criteria such as age, chronic conditions or treatment costs. In CEA systems, benefits are predominantly considered from the economic view of the society as a whole, and the individual patient's interests are more or less excluded from financial evaluations. 
The authors share many of the political and ethical concerns regarding CEA approaches, especially for cancer care, under the conditions and healthcare frameworks that prevail in LMIC.

\section{Enrollment into public insurance schemes}

In LMIC, the implementation and roll out of national cancer care services throughout the entire population suffers from various implementation barriers. The consequences are strongly related to the type/availability of healthcare insurance and affordability (accessibility). Various factors imposed by the healthcare system framework can modulate enrollment behavior into healthcare insurance schemes, which can be used intentionally by setting the appropriate incentives. While the major motivational factors that impact on enrollment are determined by the patients' perspective, other factors are also important. These include provider motivation (acceptability), healthcare accessibility (including affordable health insurance costs, and access to free or affordable drugs), and social security against unforeseen health challenges (availability). These factors are of particular importance for cancer patients. Encouragement by friends, family members and colleagues has been found to motivate subscription to public health insurances in LMIC. In addition, the type and character of benefits for insured people related to their socioeconomic status significantly influence the success of public roll-out and outreach, which is crucial for successful implementation of cancer care. Major barriers to health insurance subscription included long queues and waiting times for treatment, a perceived poor quality of drugs, and a negative attitude of service providers both in cancer care facilities and in health insurance offices. The latter point underscores the importance of incentivizing and motivating healthcare workers at all levels of health care organization.

In the context of the above-mentioned financial incentives, enrollment into public insurance schemes is stimulated by capitation schemes. Case- and procedure-based payments can motivate cancer care providers to engage in subscription schemes, but this is exquisitely influenced by the relationship between remuneration and financial expenditure. $\mathrm{P} 4 \mathrm{P}$ models tend to induce healthcare providers to implement patient selection based on risk, for example by only encouraging particular patient groups to subscribe to these models if they are economically advantageous for the providers. For example, if $\mathrm{P} 4 \mathrm{P}$ is mainly driven by procedure-based indicators, patients requiring that procedure will be targeted. By contrast, if outcome-related quality indicators are $\mathrm{P} 4 \mathrm{P}$ determinants, then patients with complex diseases are usually avoided. Vice versa, a patients' decision to subscribe to public insurance schemes can also be incentivized by targeting their coverage expectations (list of benefits), and by improving the accessibility of healthcare infrastructure and available healthcare professionals. In addition, consideration of cultural parameters when designing incentives, such as religious aspects and traditional delivery pathways, can also facilitate roll-out processes.

In summary, designing reliable incentives that achieve their desired effects is a highly challenging task in LMIC, especially for the implementation of population-based cancer care. In this context, special attention needs to be paid to establishing effective incentive frameworks for the management of cancer patients, given their vulnerability, the often life-threatening course of their disease, as well as their dependence on costly diagnosis and treatment. In view of this, we suggest that for LMIC it may be beneficial to separate cancer care from other healthcare areas with regard to financial incentives and reimbursement schemes.

\section{References}

1. Emery DW, De Brantes F (2015) Curing an ill healthcare system: transparent multilateral benefits, cost, and quality. Healthc Financ Manag. 69(2):80-83

2. Emanuel EJ, Ubel PA, Kessler JB, Meyer G, Muller RW, Navathe AS, Patel P, Pearl R, Rosenthal MB, Sacks L, Sen AP, Sherman P, Volpp KG (2016) Using behavioral economics to design physician incentives that deliver high-value care. Ann Intern Med 164(2):114-119. https://doi.org/10.7326/M15-1330

3. Liu X, Dou L, Zhang H, Sun Y, Yuan B (2015) Analysis of context factors in compulsory and incentive strategies for improving attraction and retention of health workers in rural and remote areas: a systematic review. Hum Resour Health. 13:61. https://doi. org/10.1186/s12960-015-0059-6

4. Luft HS (2015) Policy-oriented research on improved physician incentives for higher value healthcare. Health Serv Res 50(Suppl 2):2187-2215. https://doi.org/10.1111/1475-6773.12423

5. Setiawan D, Kotsopoulos N, Wilschut JC, Postma MJ, Connolly MP (2016) Assessment of the broader economic consequences of HPV prevention from a government-perspective: a fiscal analytic approach. PLoS ONE 11(8):e0160707. https://doi.org/10.1371/ journal.pone.0160707 (eCollection 2016)

Publisher's Note Springer Nature remains neutral with regard to jurisdictional claims in published maps and institutional affiliations. 